\title{
Validation and Potential Mechanisms of Red Cell Distribution Width as a Prognostic Marker in Heart Failure
}

\author{
LARRY A. ALLEN, MD, MHS ${ }^{1}$, G. MICHAEL FELKER, MD, MHS ${ }^{2}$, MANDEEP R. MEHRA, \\ MBBS $^{3}$, JUN R. CHIONG, MD, MPH ${ }^{4}$, STEPHANIE H. DUNLAP, DO ${ }^{5}$, JALAL K. GHALI, MD ${ }^{6}$, \\ DANIEL J. LENIHAN, MD ${ }^{7}$, RON M. OREN, MD ${ }^{8}$, LYNNE E. WAGONER, MD ${ }^{9}$, TODD A. \\ SCHWARTZ, DRPH ${ }^{10}$, and KIRKWOOD F. ADAMS JR, MD ${ }^{11}$ \\ ${ }^{1}$ Division of Cardiology, University of Colorado Denver, Aurora, CO \\ 2 Department of Medicine, Duke University School of Medicine, Duke Clinical Research Institute, \\ Durham, NC \\ ${ }^{3}$ Department of Medicine, University of Maryland School of Medicine, Baltimore, MD \\ ${ }^{4}$ Department of Medicine, Loma Linda University School of Medicine, Loma Linda, CA \\ ${ }^{5}$ Department of Internal Medicine, University of Cincinnati, Cincinnati, $\mathrm{OH}$ \\ ${ }^{6}$ Detroit Medical Center, Detroit, MI \\ ${ }^{7}$ Department of Cardiology, University of Texas MD Anderson Cancer Center, Houston, TX \\ ${ }^{8}$ Mercy lowa City, lowa City, IA \\ ${ }^{9}$ Greater Cincinnati Cardiovascular Consultants, Cincinnati, $\mathrm{OH}$ \\ 10 Department of Biostatistics, University of North Carolina at Chapel Hill, Chapel Hill, NC \\ 11 Department of Medicine and Radiology, University of North Carolina at Chapel Hill, Chapel Hill, \\ NC
}

\section{Abstract}

Background: Adverse outcomes have recently been linked to elevated red cell distribution width (RDW) in heart failure. Our study sought to validate the prognostic value of RDW in heart failure and to explore the potential mechanisms underlying this association.

Methods and Results: Data from the Study of Anemia in a Heart Failure Population (STAMINA-HFP) registry, a prospective, multicenter cohort of ambulatory patients with heart failure supported multivariable modeling to assess relationships between RDW and outcomes. The association between RDW and iron metabolism, inflammation, and neurohormonal activation was studied in a separate cohort of heart failure patients from the United Investigators to Evaluate Heart Failure (UNITE-HF) Biomarker registry. RDW was independently predictive of outcome (for each 1\% increase in RDW, hazard ratio for mortality 1.06, 95\% CI 1.01-1.12; hazard ratio for hospitalization or mortality 1.06; 95\% CI 1.02-1.10) after adjustment for other covariates. Increasing RDW correlated with decreasing hemoglobin, increasing interleukin-6, and impaired iron mobilization. 
Conclusions: Our results confirm previous observations that RDW is a strong, independent predictor of adverse outcome in chronic heart failure and suggest elevated RDW may indicate inflammatory stress and impaired iron mobilization. These findings encourage further research into the relationship between heart failure and the hematologic system.

\section{Keywords}

Anemia; biomarker; laboratory tests

Identification of novel markers of prognosis may improve clinical decision making, and may also provide insights into the pathophysiology of complex clinical syndromes such as heart failure. A large body of recent literature has characterized the strong relationship between anemia and adverse outcomes in heart failure. ${ }^{1-7}$ These data have led to the hypothesis that anemia may be a therapeutic target in heart failure, and have resulted in increased interest in pathophysiologic links between heart failure and the hematologic system. ${ }^{8,9}$

We recently reported the strong association of increasing red cell distribution width (RDW), a measure of the variability in size of circulating red cells, with adverse outcomes in 2 large chronic heart failure cohorts. ${ }^{10}$ This association was consistently independent of hemoglobin, and persisted as 1 of the strongest overall predictors of prognosis despite adjustment for a broad array of clinical and laboratory variables. Subsequently, RDW has also been shown to correlate with adverse outcomes in populations of patients with established coronary disease, ${ }^{11}$ those referred for coronary angiography ${ }^{12}$ in middle-aged and older adults, ${ }^{13}$ and in the general population. ${ }^{14}$ Before novel prognostic biomarkers such as RDW can be incorporated into clinical practice, they require confirmation in multiple datasets evaluating broad populations with the disease of interest to definitively establish validity and generalizability. Additionally, understanding the pathophysiology underlying the association of novel biomarkers with outcomes provides support for the biologic plausibility of the biomarker, and may provide additional insight into fundamental mechanisms of disease. Using data from 2 prospective, multicenter registries, we sought to validate our previous observations on the association between RDW and prognosis and to explore potential mechanisms underlying that association in ambulatory patients with chronic heart failure.

\section{Methods}

\section{Study Populations}

STAMINA-HFP Registry-The Study of Anemia in a Heart Failure Population (STAMINA-HFP) registry was a multicenter, prospective, observational study of a randomly selected sample of ambulatory patients with heart failure in the United States, including both academic specialty sites and community practices with an interest in heart failure. ${ }^{15}$ The heart failure specialty sites were part of the United Investigators to Evaluate Heart Failure (UNITE-HF), an investigative research group of heart failure specialists created to conduct registry and other types of clinical studies in patients with this syndrome. ${ }^{16}$ Patients were eligible for participation if they were 18 years of age or older, had symptomatic heart failure, and were willing and able to complete quarterly telephone interviews and other study assessments. Eligibility was not dependent on a specific left ventricular ejection fraction or the presence of anemia. Both new and return outpatients with heart failure were candidates for study enrollment. Patients were randomly selected for participation using an algorithm to select every $\mathrm{n}^{\text {th }}$ patient with heart failure on the clinic schedule as a potential candidate for enrollment. 
Detailed demographic, clinical, and laboratory data were collected on all patients at the time of enrollment. Hemoglobin was determined at enrollment and during follow-up clinic visits by finger-stick (HemoCue). Red cell distribution width was determined by automated complete blood cell counts from a baseline blood sample processed at each site's clinical laboratory or the most recent results available in patients lacking a baseline sample. These values were available within 30 days of enrollment in $96.4 \%$ of the patients. Follow-up clinical data were collected naturalistically from medical encounters related to usual care or as indicated by medical need.

UNITE-HF Biomarker Registry-A separate prospective multicenter registry, the UNITE-HF Biomarker registry was conducted in parallel to STAMINA-HFP, with the goal of evaluating biomarkers and clinical correlates of anemia in patients with heart failure. This study enrolled a convenience sample of outpatients with symptomatic heart failure recruited by a subgroup of the UNITE-HF investigators. Enrollment criteria were the same for both registries, but each registry recruited a distinct cohort of patients. Patients in the UNITE-HF Biomarker registry had a baseline visit to collect detailed information concerning their medical history and heart failure state. Patient follow-up was obtained through routine clinic visits and yearly contact (for at least 2 years) to determine vital status and occurrence of hospitalization.

Venous samples of whole blood, plasma, and serum were collected in the UNITE-HF Biomarker registry at the baseline clinic visit that occurred during regular daytime hours. Plasma was collected from whole blood processed in prechilled EDTA tubes by cold centrifugation $\left(4^{\circ} \mathrm{C}\right)$ at $2000 \mathrm{~g}$ within 20 minutes of collection. Plasma and serum samples were stored at $-70^{\circ} \mathrm{C}$ until they could be batch shipped under dry ice to the UNITE-HF Biomarker Core Laboratory at the University of North Carolina at Chapel Hill. A portion of the patient's whole blood (collected in an EDTAVacutainer tube) and serum were stored at $4^{\circ} \mathrm{C}$ and sent by overnight mail to the core laboratory. Subsequently, automated complete blood counts, routine chemistries, and measurements of iron status were determined on these samples by standard methods in the clinical laboratory of the University of North Carolina Hospitals. Serum levels of tumor necrosis factor alpha, interleukin (IL)-1 beta, and IL-6 were measured by high-sensitivity enzyme-linked immunosorbent assay and serum erythropoietin and plasma levels of endothelin-1 were measured by standard enzyme linked immunosorbent assay methods. All assays were conducted using commercially available kits (Quantikine, R\&D Systems, Minneapolis, MN). The minimum detectable concentration of tumor necrosis factor alpha was $<4.4 \mathrm{pg} / \mathrm{mL}$, whereas for IL-1 beta and IL-6 it was $<1 \mathrm{pg}$ / $\mathrm{mL}$. Assays were performed by technicians unaware of patient clinical characteristics and outcome.

Both protocols were approved by the relevant local institutional review boards, and all enrolled patients provided written, informed consent.

\section{Statistical Analysis}

STAMINA-HFP Registry-The primary study analysis used Cox proportional hazards models, univariable and multivariable, to evaluate the association between RDW (as a continuous variable) and the clinical end points of all-cause mortality and all-cause hospitalization or mortality. Variables considered for inclusion in the multivariable models as potential confounders of the relationship of RDW to study outcomes were: gender; race; age; New York Heart Association functional class; estimated glomerular filtration rate; history of diabetes, hypertension, atrial fibrillation or flutter; left ventricular ejection fraction; ischemic heart disease; systolic blood pressure; diastolic blood pressure; heart rate; pulse pressure; edema by physical exam; or current smoking and current medications 
(angiotensin-converting enzyme inhibitor, angiotensin receptor blocker, $\beta$-blocker, any diuretic, loop diuretic). Each was assessed univariably with the study outcomes, and those with $P<.05$ were included in multivariable models, through which adjusted hazard ratios for RDW were determined. Because of high levels of missing values, duration of heart failure, body mass index, and blood urea nitrogen were also considered for subsequent inclusion to supplemental multivariable models where applicable. The relationships between the variable of interest (RDW) and the outcomes were expressed using hazards ratios (HR) and their 95\% confidence intervals (CI), and the relative strength of association of each predictor was assessed based on the chi-square statistic with 1 degree of freedom in the adjusted model. A test of the proportional hazard assumption for the RDW effect was conducted through a test of the interaction of RDW with follow-up time. Departure from linearity for the RDW effect was assessed through the joint testing of cubic and quadratic polynomial terms.

C-statistics were computed to reflect a particular model's degree of discrimination for whether a patient has an event or not. Such models were formulated with and without RDW as a predictor, and the corresponding c-statistics indicate the additional discrimination provided by inclusion of RDW in the model.

For descriptive purposes, patients in the study cohort from the STAMINA-HFP registry $(\mathrm{n}=$ 1016) were grouped into ranges of RDW based on the lowest quartile of RDW, middle 50\% range of RDW, and the highest quartile of RDW. The distribution of RDW values was also characterized based on the normal range defined as $₫ 5 \%$. Kaplan Meier methods were used to examine unadjusted event rates at 1 year, and the log-rank test assessed differences among event rates across the RDW ranges and $P$ values for differences among these patient groups. Differences in baseline characteristics across these same groups were determined using analysis of variance and chi-square tests, as appropriate.

UNITE-HF Biomarker Registry-Spearman correlation analysis examined the general relationships between RDW and biomarkers of inflammation, iron metabolism, and neurohormonal activation. For descriptive purposes, patients in the UNITE-HF Biomarker registry were also grouped based on the same cut points of RDW used in the STAMINAHFP registry. Biomarkers of interest were evaluated across these 2 patient groups by trend testing using Spearman correlation (with results reported through medians and first and third quartiles). Recognizing that selection of the RDW grouping cut points above was arbitrary, additional descriptive analyses were performed with patients divided into normal versus abnormal RDW groups with the normal range for RDW defined as $\unlhd 5 \%$, as in the STAMINA-HFP registry. Differences between these groups were assessed by $t$-tests and chi-square tests as appropriate.

Data on various inflammatory cytokines and endothelin-1 were available in subsets of the cohort with RDW. To help address the potential of bias in the selection of these patients, baseline characteristics in the IL-6 subset were compared with the overall cohort with RDW. Characteristics that differed were included in multiple linear regression modeling of the relationship of IL-6 to RDW.

\section{General Considerations}

Results summarizing the baseline characteristics of patients in both registries are reported as means \pm standard deviations (SD), medians, first and third quartiles (Q1 and Q3, respectively), or percentages as appropriate. In this report, anemia was defined using the World Health Organization criteria of baseline hemoglobin $<13 \mathrm{~g} / \mathrm{dL}$ for men and $<12 \mathrm{~g} / \mathrm{dL}$ for women. ${ }^{17} \mathrm{~A} P$ value $<.05$ was considered statistically significant in all analyses. Analyses were conducted with SAS 9.1 (SAS Institute, Inc, Cary, NC). 
The authors had full access to the data and take responsibility for its integrity. All authors have read and agree to the manuscript as written.

\section{Results}

\section{STAMINA-HFP Registry}

Baseline Characteristics-A total of 1016 (94\%) of 1082 subjects in the STAMINAHFP registry had RDW values available and formed the population for this analysis. This cohort was older (37\% age $\geq 70$ ), male, and Caucasian (Table 1). Thirty-four percent of patients had anemia based on World Health Organization criteria. For this registry cohort, the mean RDW was $14.7 \pm 2.5 \%$. The median RDW in the cohort was $14.2 \%(\mathrm{Q} 1-\mathrm{Q} 3$ $13.4-15.5 \%$ ), and $31 \%$ of patients had an abnormal value (> 15\%). RDW was modestly correlated with hemoglobin (Spearman $r=-0.24, P<.001$ ). In general, patients with elevated RDW were older, more likely to be African American, and tended to have more severe heart failure and a greater burden of comorbidity, as shown in Table 1.

RDW and Clinical Outcomes-Data on outcomes were available in 1012 patients in the STAMINA-HFP study cohort. The mean duration of follow-up of these patients was $1.0 \pm$ 0.3 years. There were 77 deaths during follow-up, and the crude 1-year mortality rate was $7.5 \%$ (Table 2). Two hundred ninety-two patients had at least 1 all-cause hospitalization during follow-up (29\% of the cohort with hospitalization data).

Crude clinical outcomes for RDW ranges are shown in Table 2, and Kaplan Meier curves stratified by RDW ranges are shown in Fig. 1. In univariate analysis, RDW was strongly associated with adverse outcome, both for all-cause mortality (unadjusted HR 1.09 per 1\% increase in RDW, 95\% CI 1.06 to $1.13, P<.001$ ) and for the composite of all-cause hospitalization or mortality (unadjusted HR 1.10 per 1\% increase in RDW, 95\% CI 1.07 to $1.13, P<.001)$. Based on the global chi-square statistic in the final adjusted model, elevated RDW had the third strongest statistical association with all-cause mortality (Table 3 ) and the combined end point of all-cause hospitalization or mortality (Table 4) among the variables modeled. Notably, RDW was a stronger predictor of mortality than many other wellvalidated predictors, including functional class and age. RDW was found to be a significant independent predictor of all-cause mortality (adjusted HR 1.07 per $1 \%$ increase in RDW, 95\% CI 1.01 to $1.13, P=.018$ ) and all-cause hospitalization or mortality (adjusted HR 1.05, $95 \%$ CI 1.01 to $1.10, P=.025$ ) in supplemental modeling that included adjustment for blood urea nitrogen.

No significant evidence was found for a violation of the proportional hazards assumption in the mortality model $(P=.32)$, nor was there significant evidence for a nonlinear effect of RDW on the mortality outcome $(P=.35)$.

C-statistic Analysis-Results reflecting the evaluation of RDW and other clinical predictors for the mortality end point using the c-statistic are shown in Table 5. RDW and estimated glomerular filtration rate alone were found to have the highest c-statistic of any of the significant predictors determined by multivariable modeling of mortality. The c-statistic of the final clinical model lacking RDW did not increase significantly when RDW was added.

\section{UNITE-HF Biomarker Registry}

Baseline Characteristics-The UNITE-HF Biomarker registry enrolled a total of 291 patients who had baseline clinical data and blood available for biomarker analysis. In the current study, results are reported from 235 of these 291 patients who had RDW values 
available from the registry clinical core laboratory. As expected, the baseline characteristics of these patients differed in a number of ways compared with the unselected study patients from the STAMINA-HFP registry. UNITE-HF Biomarker patients were younger, more likely to be male and African American (data not shown). Median RDW in the UNITE-HF Biomarker cohort was $14.0 \%$ (Q1-Q3 13.2-15.3\%). Mean age was $59 \pm 14$ years, $29 \%$ were female, with a mean ejection fraction $32 \pm 15 \%$, New York Heart Association functional class of $2.6 \pm 0.9$, and estimated glomerular filtration rate of $67 \pm 25 \mathrm{~mL} \cdot \mathrm{min} \cdot 1.73 \mathrm{~m}^{2}$. The mean hemoglobin of the UNITE-HF Biomarker cohort was $13.5 \pm 1.7 \mathrm{~g} / \mathrm{dL}$ with $31 \%$ having anemia by World Health Organization criteria.

Association of RDW with Inflammation and Iron Status-The relationship between RDW and iron studies, inflammatory cytokines, and other heart failure biomarkers are shown in Tables 6 and 7. These tables show the results of both continuous analyses of various measures with RDW and descriptive, categorical analyses based on RDW patient groups. Increasing RDW was associated with a biochemical profile suggestive of impaired iron mobilization, typical of anemia of chronic disease. As RDW increased, mean corpuscular volume decreased, suggesting that the increased variability of red cell size in the highest RDW patients was due to a greater number of small red blood cells (Fig. 2A). The classical definition of iron deficiency (iron binding saturation $<20 \%$ ) was present in $46 \%$ of patients. Serum iron levels decreased at higher RDW levels, whereas iron binding capacity remained stable. As a result, iron saturation decreased with increasing RDW (Fig. 2B). Consistent with a state of chronic inflammation, ferritin levels were normal in all groups regardless of RDW. Erythropoietin levels increased significantly with increasing RDW. The results suggested a specific association between the inflammatory cytokines studied and RDW. IL-6 was significantly elevated in patients with higher RDW (Table 7, Fig. 2C), whereas tumor necrosis factor-alpha did not correlate with RDW, and IL-1 beta levels were inversely correlated with RDW values. Results were similar when patients were divided into RDW groups based on the normal range for this measurement (data not shown).

This report focuses on the subset of UNITE-HF Biomarker registry patients with both RDW values and data available for analysis of hematological parameters and selected neurohormones. In the case of iron status and other hematologic indices, patients with results available were considered to be representative because these values were available in the great majority of patients in the UNITE-HF Biomarker cohort with RDW. In the case of various inflammatory cytokines and endothelin-1, results were available in smaller subsets of the study cohort. Because IL- 6 was found to be related to RDW, the 138 patients with this data were taken as an example group for comparison of baseline characteristics between patients with and without neurohormonal results in the study cohort. In addition, these patients were used to investigate the potential impact that differences in baseline characteristic may have had on the reported associations between RDW and various neurohormones. IL- 6 data were deemed representative of anemic patients in the study cohort because IL-6 values were available in 68 of the 72 anemic study patients. In contrast, IL-6 results were available in a smaller percentage of nonanemic patients (69 of 162) and there were a number of differences in baseline characteristics between nonanemic patients with and without these results (data not shown). However, of the baseline characteristics that differed between nonanemics with and without IL-6 results, only heart rate was found to be a significant predictor of values of this cytokine (data not shown). Importantly, RDW was significantly related to IL-6 (adjusted $P<.001$ ) after taking into account heart rate as well as all other characteristics that differed between nonanemic patients with and without IL-6 values. 


\section{Discussion}

In this study, we confirmed our previous report of RDW as a powerful marker of prognosis in chronic heart failure. RDW was among the strongest predictors of both mortality and hospitalization, even after adjustment for a variety of other clinical and laboratory variables. As evaluated by the chi-square statistic in the final multivariable model, the prognostic significance of RDW was comparable to other widely accepted risk markers such as ejection fraction and New York Heart Association class. The adjusted standardized HR for mortality (per 1 standard deviation of RDW change) in the STAMINA-HFP registry was 1.17 (95\% CI 1.03 to 1.32), which was similar to that seen in the Candesartan in Heart Failure Assessment of Reduction in Mortality and Morbidity (CHARM) Program (1.12) and the Duke Databank (1.29). ${ }^{10} \mathrm{C}$-statistic results also pointed to the importance of RDW relative to other independent predictors of mortality in our study population. Additionally, the current study provides the first data evaluating the relationship between RDW and biomarkers of inflammation and iron metabolism in heart failure. We found that higher RDW was associated with biochemical evidence of greater inflammation and impaired iron mobilization, suggesting that elevated RDW may be an integrative measure of several potentially important pathophysiologic processes in heart failure.

\section{Importance of RDW as a Risk Predictor}

Although potential markers of risk are frequently identified using multivariable modeling in large datasets, often little effort is made to replicate these findings in independent cohorts, thereby limiting the validity and generalizability of the observation. Elevated RDW has now been shown to be a robust marker of risk in 3 distinct cohorts of chronic heart failure, including a large multicenter clinical trial, a single center registry focused on patients undergoing cardiac catheterization, and a multicenter, unselected ambulatory heart failure population. Notably, all 3 of these cohorts include patients with both impaired and preserved ejection fraction, and were thus broadly representative of the epidemiology of chronic heart failure. The consistency of these findings across multiple independent datasets argues strongly for the validity of this association with adverse outcomes. In addition to its strong association with mortality and hospitalization, RDW is routinely available as part of the automated blood count that is a standard component routine medical care. Thus, RDW may represent an exception to the adage that "there's no such thing as a free lunch," providing significant prognostic information without adding cost or complexity to current heart failure management.

\section{Potential Mechanisms}

An emerging concept in cardiovascular disease is the role of the erythrocyte as a barometer of overall cardiovascular health. ${ }^{18} \mathrm{~A}$ variety of mechanisms have been posited to explain the association between anemia and outcomes in heart failure, including inflammatory stress, nutritional deficiencies, inadequate production of erythropoietin, and the impact of comorbidities. 6,9 Any or all of these mechanisms can result in anemia, and each may also lead to elevations in RDW. RDW is a quantitative measure of the variability in size of the circulating erythrocytes (ie, anisocytosis). Classically, RDW is elevated in conditions of ineffective red cell production (eg, iron deficiency, anemia of chronic disease, B12 or folate deficiency, and hemoglobinopathies), increased red cell destruction (eg, hemolysis), or after blood transfusion.

The association of elevated RDW with low hemoglobin, moderately depressed mean corpuscular volume, high erythropoietin, normal iron binding capacity, and normal ferritin are all consistent with a state of impaired iron mobilization. This results in inhibition of erythropoietin-induced erythrocyte maturation, the hallmark of anemia of chronic disease. ${ }^{19}$ 
Although the prevalence of frank iron deficiency in patients with heart failure is controversial, there is increasing recognition that the ability to mobilize and use existing iron stores may be impaired even in the setting of adequate total body iron. ${ }^{20-22}$ This so called "reticuloendothelial block" is mediated in part by overexpression of hepcidin, a peptide hormone secreted by the liver which acts as a regulator of human iron metabolism. ${ }^{23} \mathrm{By}$ decreasing cell surface expression of the iron exporter ferroportin, hepcidin decreases iron absorption from the intestine and iron release from reticuloendothelial stores. Hepcidin is upregulated by a number of stimuli, including anemia, hypoxia, and, in particular, inflammation. ${ }^{24,25} \mathrm{IL}-6$, which was strongly associated with elevated RDW in our study, is a powerful inducer of transcription of the hepcidin gene. ${ }^{26}$ Additionally, inflammatory cytokines may directly inhibit erythropoietin-induced erythrocyte maturation, which is reflected by an increase in RDW. ${ }^{27,28}$ Taken as a whole, our data suggest the hypothesis that elevations in RDW may reflect a variety of underlying pathologic processes, such as inflammatory stress and impaired iron metabolism that may directly contribute to disease progression in heart failure. Given the challenges of assessing these underlying processes in clinical practice, RDW may provide a widely available, inexpensive "barometer" that integrates these complex interactions into a single, well-validated prognostic biomarker.

\section{Limitations}

Although the sample size of the current study was large, the mean follow-up was relatively short at 1 year, which limited the number of overall events. As with all analyses of observational data, our study cannot distinguish causality from association, both with regard to the link between RDW and outcomes and between RDW, inflammation, and iron metabolism. Patients in the STAMINA-HFP registry differ from those in the biomarker cohort in a number of ways that make it difficult to link findings on outcomes and disordered iron metabolism from 1 registry to the other. Biochemical data on iron metabolism and inflammatory cytokines were available on only a subset of the UNITE-HF Biomarker registry patients. Although this bias could have influenced the relationships observed between these measures and RDW, as an example, modeling results with IL-6 still indicate a significant relationship between this cytokine and RDW after accounting for differences observed.

\section{Conclusions}

Elevated RDW had a strong, independent association with adverse outcomes in this unselected, broadly representative cohort of ambulatory heart failure patients. RDW was a more powerful predictor of outcome than many commonly used predictors, and has the advantage of already being routinely available as part of the automated complete blood count. Elevations in RDW appear to reflect a state of increased inflammation and impaired iron metabolism, findings that suggest the possibility that RDW may provide an integrated measure of these underlying processes in heart failure. These findings encourage ongoing research into the relationship between the hematologic system and human heart failure.

\section{Acknowledgments}

The authors appreciate the tireless persistence and exacting thoroughness of Amanda Garrand that was essential to the successful preparation of this manuscript.

Supported by the STAMINA-HFP and UNITE-HF Biomarker registries were funded by Amgen, Inc (Thousand Oaks, CA). 


\section{References}

1. Al-Ahmad A, Rand WM, Manjunath G, Konstam MA, Salem DN, Levey AS, et al. Reduced kidney function and anemia as risk factors for mortality in patients with left ventricular dysfunction. J Am Coll Cardiol. 2001; 38:955-62. [PubMed: 11583864]

2. Horwich TB, Fonarow GC, Hamilton MA, MacLellan WR, Borenstein J. Anemia is associated with worse symptoms, greater impairment in functional capacity and a significant increase in mortality in patients with advanced heart failure. J Am Coll Cardiol. 2002; 39:1780-6. [PubMed: 12039491]

3. Mozaffarian D, Nye R, Levy WC. Anemia predicts mortality in severe heart failure: the prospective randomized amlodipine survival evaluation (PRAISE). J Am Coll Cardiol. 2003; 41:1933-9. [PubMed: 12798560]

4. Ezekowitz JA, McAlister FA, Armstrong PW. Anemia is common in heart failure and is associated with poor outcomes: insights from a cohort of 12065 patients with new-onset heart failure.

Circulation. 2003; 107:223-5. [PubMed: 12538418]

5. Felker GM, Gattis WA, Leimberger JD, Adams KF, Cuffe MS, Gheorghiade M, et al. Usefulness of anemia as a predictor of death and rehospitalization in patients with decompensated heart failure. Am J Cardiol. 2003; 92:625-8. [PubMed: 12943893]

6. Tang YD, Katz SD. Anemia in chronic heart failure: prevalence, etiology, clinical correlates, and treatment options. Circulation. 2006; 113:2454-61. [PubMed: 16717164]

7. Ghali JK. Anemia and heart failure. Curr Opin Cardiol. 2009; 24:172-8. [PubMed: 19532104]

8. van Veldhuisen DJ, McMurray JJ. Are erythropoietin stimulating proteins safe and efficacious in heart failure? Why we need an adequately powered randomised outcome trial. Eur J Heart Fail. 2007; 9:110-2. [PubMed: 17270493]

9. Felker GM, Adams KF Jr, Gattis WA, O'Connor CM. Anemia as a risk factor and therapeutic target in heart failure. J Am Coll Cardiol. 2004; 44:959-66. [PubMed: 15337204]

10. Felker GM, Allen LA, Pocock SJ, Shaw LK, McMurray JJ, Pfeffer MA, et al. Red cell distribution width as a novel prognostic marker in heart failure: data from the CHARM Program and the Duke Databank. J Am Coll Cardiol. 2007; 50:40-7. [PubMed: 17601544]

11. Tonelli M, Sacks F, Arnold M, Moye L, Davis B, Pfeffer M. Relation between red blood cell distribution width and cardiovascular event rate in people with coronary disease. Circulation. 2008; 117:163-8. [PubMed: 18172029]

12. Cavusoglu E, Chopra V, Gupta A, Battala VR, Poludasu S, Eng C, et al. Relation between red blood cell distribution width (RDW) and all-cause mortality at two years in an unselected population referred for coronary angiography. Int J Cardiol. 2009 article in press.

13. Patel KV, Ferrucci L, Ershler WB, Longo DL, Guralnik JM. Red blood cell distribution width and the risk of death in middle aged and older adults. Arch Intern Med. 2009; 169:515-23. [PubMed: 19273783]

14. Perlstein TS, Weuve J, Pfeffer MA, Beckman JA. Red blood cell distribution width and mortality risk in a community-based prospective cohort. Arch Intern Med. 2009; 169:588-94. [PubMed: 19307522]

15. Adams KF Jr, Patterson JH, Oren RM, Mehra MR, O'Connor CM, Pina IL, et al. Prospective assessment of the occurrence of anemia in patients with heart failure: results from the Study of Anemia in a Heart Failure Population (STAMINA-HFP) Registry. Am Heart J. 2009; 157:926-32. [PubMed: 19376323]

16. Adams KF, O’Connor CM, Oren RM, Ventura HO, Pina I, Gattis WA, et al. Development of a multicenter heart failure database: initial report from the United Investigators to Evaluate Heart Failure [abstract]. J Card Fail. 2000; 6:S2-56.

17. World Health Organization. Iron deficiency anemia, assessment, prevention, and control: report of a WHO Scientific Group. WHO; Geneva, Switzerland: 2001.

18. Minetti M, Agati L, Malorni W. The microenvironment can shift erythrocytes from a friendly to a harmful behavior: pathogenetic implications for vascular diseases. Cardiovasc Res. 2007; 75:21-8. [PubMed: 17412313]

19. Weiss G, Goodnough LT. Anemia of chronic disease. N Engl J Med. 2005; 352:1011-23.

[PubMed: 15758012] 
20. Opasich C, Cazzola M, Scelsi L, De Feo S, Bosimini E, Lagioia R, et al. Blunted erythropoietin production and defective iron supply for erythropoiesis as major causes of anaemia in patients with chronic heart failure. Eur Heart J. 2005; 26:2232-7. [PubMed: 15987710]

21. Nanas JN, Matsouka C, Karageorgopoulos D, Leonti A, Tsolakis E, Drakos SG, et al. Etiology of anemia in patients with advanced heart failure. J Am Coll Cardiol. 2006; 48:2485-9. [PubMed: 17174186]

22. Westenbrink BD, Voors AA, van Veldhuisen DJ. Is anemia in chronic heart failure caused by iron deficiency? J Am Coll Cardiol. 2007; 49:2301-2. [PubMed: 17560297]

23. Ganz T. Hepcidin, a key regulator of iron metabolism and mediator of anemia of inflammation. Blood. 2003; 102:783-8. [PubMed: 12663437]

24. De Domenico I, Ward DM, Kaplan J. Hepcidin regulation: ironing out the details. J Clin Invest. 2007; 117:1755-8. [PubMed: 17607352]

25. Nicolas G, Chauvet C, Viatte L, Danan JL, Bigard X, Devaux I, et al. The gene encoding the iron regulatory peptide hepcidin is regulated by anemia, hypoxia, and inflammation. J Clin Invest. 2002; 110:1037-44. [PubMed: 12370282]

26. Lee P, Peng H, Gelbart T, Wang L, Beutler E. Regulation of hepcidin transcription by interleukin-1 and interleukin-6. Proc Natl Acad Sci USA. 2005; 102:1906-10. [PubMed: 15684062]

27. Chiari MM, Bagnoli R, De Luca PD, Monti M, Rampoldi E, Cunietti E. Influence of acute inflammation on iron and nutritional status indexes in older inpatients. J Am Geriatr Soc. 1995; 43:767-71. [PubMed: 7602028]

28. Pierce CN, Larson DF. Inflammatory cytokine inhibition of erythropoiesis in patients implanted with a mechanical circulatory assist device. Perfusion. 2005; 20:83-90. [PubMed: 15918445] 

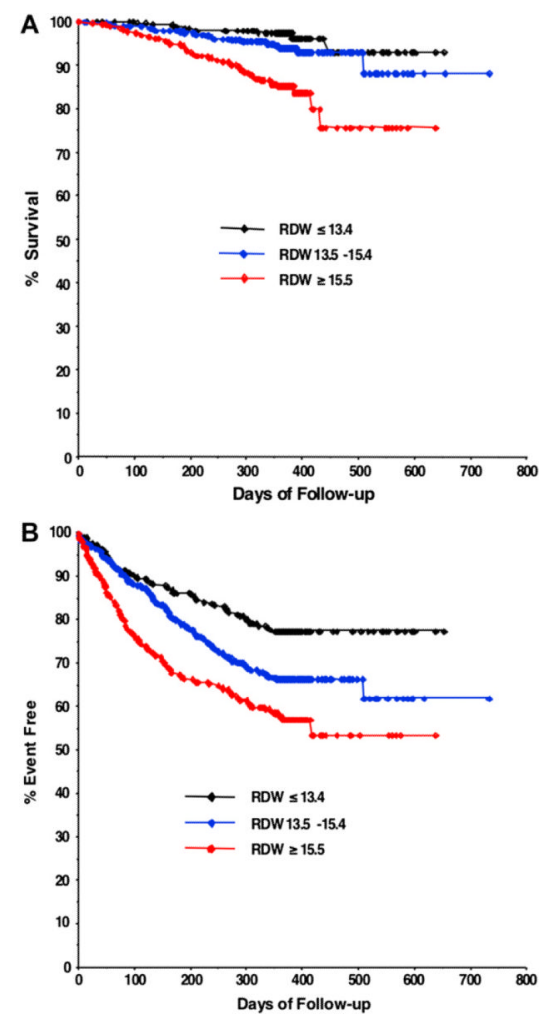

Fig. 1.

Unadjusted relationship of red cell distribution width to ad verse outcomes. Kaplan Meier curves for all-cause mortality (A) and hospitalization or mortality (B) by ranges of red cell distribution width. 

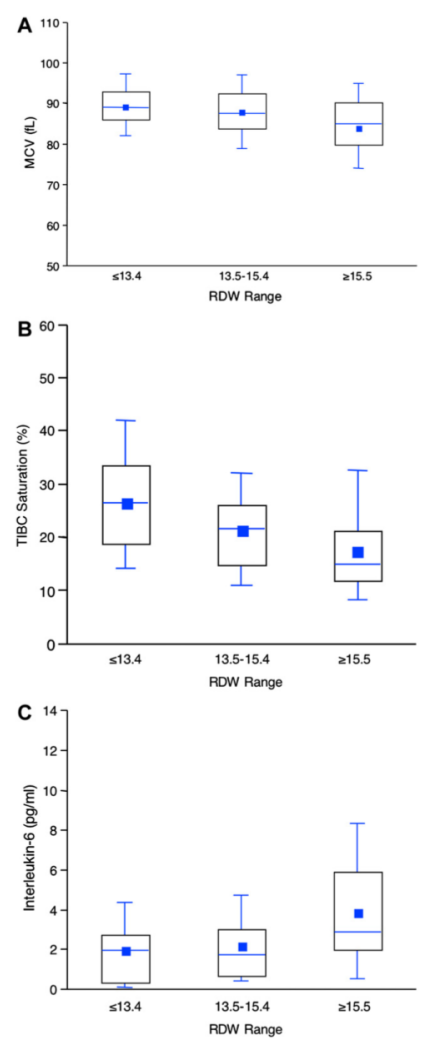

Fig. 2.

Relationship of red cell distribution width to indices of iron metabolism and inflammation. Association of ranges of red cell distribution width with mean corpuscular volume (A), total iron binding capacity (TIBC) saturation (B), and interleukin (IL)-6 (C). Boxes illustrate the 25 th, 50th, and 75 th percentiles of the distribution. The mean is given by a square, the median by the horizontal line, and the whiskers extend to the 5th and 95th percentiles. 
Table 1

Baseline Characteristics for the STAMINA-HFP Registry across Ranges of Red Cell Distribution Width

\begin{tabular}{|c|c|c|c|c|c|}
\hline Characteristic & All Patients & $\mathbf{4 3 . 4 \%}$ & $\begin{array}{c}\text { RDW Ranges } \\
\mathbf{1 3 . 5 - 1 5 . 4 \%}\end{array}$ & $\geq 15.5 \%$ & $P$ \\
\hline $\mathrm{n}$ & 1016 & 265 & 493 & 258 & \\
\hline RDW (\%) & $14.7 \pm 2.5$ & $12.8 \pm 0.5$ & $14.3 \pm 0.6$ & $17.5 \pm 3.5$ & $<.001$ \\
\hline \multicolumn{6}{|l|}{ Demographics } \\
\hline Age (y) & $64 \pm 14$ & $61 \pm 15$ & $64 \pm 14$ & $66 \pm 14$ & .003 \\
\hline Gender ( $\%$ female) & 42 & 45 & 43 & 38 & .237 \\
\hline Race (\% Caucasian) & 73 & 78 & 73 & 68 & .030 \\
\hline \multicolumn{6}{|l|}{ Symptoms } \\
\hline Mean NYHA class & $2.2 \pm 0.7$ & $2.1 \pm 0.7$ & $2.2 \pm 0.7$ & $2.5 \pm 0.8$ & $<.001$ \\
\hline Class I (\%) & 15 & 21 & 15 & 11 & $<.001$ \\
\hline Class II (\%) & 47 & 54 & 48 & 37 & \\
\hline Class III (\%) & 35 & 24 & 37 & 45 & \\
\hline Class IV (\%) & 2 & 1 & 1 & 7 & \\
\hline \multicolumn{6}{|l|}{ Medical history } \\
\hline $\operatorname{IHD}(\%)$ & 50 & 45 & 49 & 55 & .082 \\
\hline Hypertension (\%) & 69 & 66 & 70 & 71 & .326 \\
\hline $\begin{array}{l}\text { Atrial } \\
\quad \text { fibrillation-flutter (\%) }\end{array}$ & 31 & 22 & 29 & 42 & $<.001$ \\
\hline Diabetes (\%) & 38 & 30 & 39 & 43 & .004 \\
\hline Current smoking (\%) & 11 & 11 & 11 & 10 & .866 \\
\hline Current alcohol (\%) & 21 & 25 & 21 & 17 & .080 \\
\hline \multicolumn{6}{|l|}{ Physical exam } \\
\hline Edema $(\%)$ & 34 & 25 & 34 & 43 & $<0.001$ \\
\hline Rales (\%) & 6 & 6 & 5 & 8 & .328 \\
\hline $\operatorname{JVD}(\%)$ & 12 & 10 & 12 & 15 & .133 \\
\hline $\mathrm{SBP}(\mathrm{mm} \mathrm{Hg})$ & $122 \pm 21$ & $122 \pm 19$ & $123 \pm 22$ & $121 \pm 20$ & .351 \\
\hline $\mathrm{DBP}(\mathrm{mm} \mathrm{Hg})$ & $71 \pm 12$ & $71 \pm 11$ & $71 \pm 12$ & $70 \pm 12$ & .559 \\
\hline HR (beats/min) & $73 \pm 12$ & $72 \pm 12$ & $73 \pm 12$ & $74 \pm 12$ & .320 \\
\hline \multicolumn{6}{|l|}{ Current medications } \\
\hline$\beta$-blocker (\%) & 81 & 83 & 80 & 80 & .463 \\
\hline ACEI or ARB (\%) & 87 & 90 & 87 & 84 & .112 \\
\hline Digoxin $(\%)$ & 47 & 43 & 48 & 50 & .196 \\
\hline Loop diuretic (\%) & 80 & 72 & 81 & 88 & $<.001$ \\
\hline \multicolumn{6}{|l|}{ Laboratory data } \\
\hline Creatinine (mg/dL) & $1.4 \pm 1.1$ & $1.2 \pm 0.9$ & $1.4 \pm 1.3$ & $1.6 \pm 1.0$ & .003 \\
\hline eGFR (mL·min $\left.1.73 \mathrm{~m}^{2}\right)$ & $64 \pm 26$ & $69 \pm 24$ & $64 \pm 26$ & $58 \pm 27$ & $<.001$ \\
\hline Hemoglobin (g/dL) & $13.3 \pm 2.1$ & $13.8 \pm 1.9$ & $13.4 \pm 2.0$ & $12.5 \pm 2.1$ & $<.001$ \\
\hline $\operatorname{LVEF}(\%)$ & $35 \pm 16$ & $36 \pm 16$ & $36 \pm 16$ & $33 \pm 16$ & .081 \\
\hline
\end{tabular}

ACEI, angiotensin-converting enzyme inhibitor; ARB, angiotensin II receptor blocker; DBP, diastolic blood pressure; eGFR, estimated glomerular filtration rate; HR, heart rate; IHD, ischemic heart disease; JVD, jugular venous distention; LVEF, left ventricular ejection fraction; NYHA, New 
York Heart Association; RDW, red cell distribution width; SBP, systolic blood pressure; STAMINA-HFP, Study of Anemia in a Heart Failure Population.

Continuous variables are expressed as means \pm standard deviation. $P$ values are from analyses of variance or chi-square tests for differences among the groups. 
Table 2

Clinical Outcomes for the STAMINA-HFP Registry at 1 Year across Ranges of Red Cell Distribution Width

\begin{tabular}{lccccc}
\hline Outcome & All Patients & $\mathbf{4 3 . 4 \%}$ & $\begin{array}{c}\text { RDW Ranges } \\
\mathbf{1 3 . 5 - 1 5 . 5 \%}\end{array}$ & $\mathbf{\mathbf { 1 5 . 5 \% }}$ & $\boldsymbol{P}$ \\
\hline $\mathrm{n}$ & 1012 & 264 & 491 & 257 & \\
All-cause mortality & $7.5(77)$ & $2.5(8)$ & $6.2(30)$ & $15(39)$ & $<.001$ \\
At least 1 all-cause hospitalization & $31(292)$ & $21(51)$ & $32(147)$ & $38(94)$ & $<.001$ \\
Death + hospitalization composite & $33(319)$ & $23(55)$ & $34(157)$ & $43(107)$ & $<.001$ \\
\hline
\end{tabular}

RDW, red cell distribution width; STAMINA-HFP, Study of Anemia in a Heart Failure Population.

Results displayed as percentages with number of events in parenthesis.

$P$ values are from the log-rank test for differences among the groups. 
Table 3

Multivariable Proportional Hazard Regression Analysis of Mortality

\begin{tabular}{lcccc}
\hline Variable & Chi-square & HR & $\mathbf{9 5 \% ~ C I ~}$ & $\begin{array}{c}\boldsymbol{P} \\
\text { Value }\end{array}$ \\
\hline RDW (per 1\%) & 6.1 & 1.06 & $1.01-1.12$ & .013 \\
$\begin{array}{l}\text { Hemoglobin } \\
\quad \text { per 1 g/dL decrease) }\end{array}$ & 1.9 & 1.09 & $0.96-1.23$ & .173 \\
Age (per 10 y) & 0.6 & 1.09 & $0.87-1.37$ & .434 \\
NYHA class at baseline & 1.7 & 1.26 & $0.89-1.77$ & .197 \\
Ischemic heart disease & $<0.1$ & 1.01 & $0.60-1.69$ & .976 \\
Hypertension & 1.8 & 1.52 & $0.83-2.77$ & .175 \\
LVEF (per 5 units) & 12.5 & 0.84 & $0.77-0.93$ & $<.001$ \\
eGFR (per 10 units) & 7.0 & 0.85 & $0.75-0.96$ & .008 \\
SBP (per 5 units) & 0.3 & 1.02 & $0.95-1.10$ & .572 \\
DBP (per 5 units) & 6.1 & 0.85 & $0.74-0.97$ & .014 \\
Atrial fibrillation & 1.6 & 1.38 & $0.84-2.27$ & .210 \\
$\quad$ or flutter & & & & .110 \\
Edema on exam & 2.6 & 1.51 & $0.91-2.51$ & .563 \\
Any diuretic & 0.3 & 0.52 & $0.06-4.73$ & .287 \\
\hline Loop diuretic & 1.1 & 2.96 & $0.40-21.87$ & \\
\hline
\end{tabular}

DBP, diastolic blood pressure; eGFR, estimated glomerular filtration rate; LVEF, left ventricular ejection fraction; NYHA, New York Heart Association; RDW, red cell distribution width; SBP, systolic blood pressure.

Chi-squared statistic each with $1 \mathrm{df}$. 
Table 4

Multivariable Proportional Hazard Regression Analysis of Combined End Point

\begin{tabular}{lcccc}
\hline Variable & Chi-square & HR & $\mathbf{9 5 \% ~ C I ~}$ & $\begin{array}{c}\boldsymbol{P} \\
\text { Value }\end{array}$ \\
\hline RDW (per 1\%) & 9.2 & 1.06 & $1.02-1.10$ & .002 \\
$\begin{array}{l}\text { Hemoglobin } \\
\quad \text { per 1 g/dL decrease) }\end{array}$ & 2.9 & 1.05 & $0.99-1.12$ & .087 \\
NYHA class at baseline & 20.0 & 1.49 & $1.25-1.77$ & $<.001$ \\
Ischemic heart disease & 2.1 & 1.20 & $0.94-1.54$ & .143 \\
Diabetes mellitus & 3.4 & 1.26 & $0.99-1.60$ & .064 \\
LVEF (per 5 units) & 8.0 & 0.94 & $0.90-0.98$ & .005 \\
eGFR (per 10 units) & 12.7 & 0.91 & $0.86-0.96$ & $<.001$ \\
SBP (per 5 units) & 0.3 & 0.99 & $0.96-1.02$ & .596 \\
Atrial fibrillation & 1.7 & 1.19 & $0.92-1.53$ & .191 \\
$\quad$ or flutter & & & & .384 \\
Edema on exam & 0.8 & 1.12 & $0.87-1.43$ & .384 \\
Any diuretic & 0.2 & 0.84 & $0.43-1.65$ & .621 \\
Loop diuretic & 0.3 & 1.19 & $0.66-2.15$ & .569 \\
\hline
\end{tabular}

eGFR, estimated glomerular filtration rate; LVEF, left ventricular ejection fraction; NYHA, New York Heart Association; RDW, red cell distribution width; SBP, systolic blood pressure.

Chi-squared statistic each with $1 \mathrm{df}$. 
Table 5

C-statistics for Independent Predictors of Mortality with and without RDW

\begin{tabular}{lcccccc}
\hline & \multicolumn{2}{c}{ Mortality } & & \multicolumn{2}{c}{$\begin{array}{c}\text { Incremental Value } \\
\text { of Adding RDW }\end{array}$} \\
\cline { 2 - 3 } \cline { 5 - 6 } & c-statistic & $\mathbf{9 5 \%} \mathbf{~ C I}$ & & c-statistic & $\mathbf{9 5 \%} \mathbf{~ C I}$ \\
\hline Independent predictors & 0.69 & $0.63-0.75$ & & NA & NA \\
RDW & 0.69 & $0.63-0.75$ & & 0.71 & $0.65-0.77$ \\
eGFR & 0.66 & $0.60-0.73$ & & 0.72 & $0.66-0.78$ \\
DBP & 0.62 & $0.55-0.69$ & & 0.68 & $0.61-0.74$ \\
LVEF & & & & & \\
Multivariable models & & & & & & \\
Overall clinical model & 0.79 & $0.73-0.84$ & & 0.79 & $0.74-0.84$
\end{tabular}

Overall clinical model characteristics same as in Table 3.

All abbreviations are the same as in Tables 1 and 3 


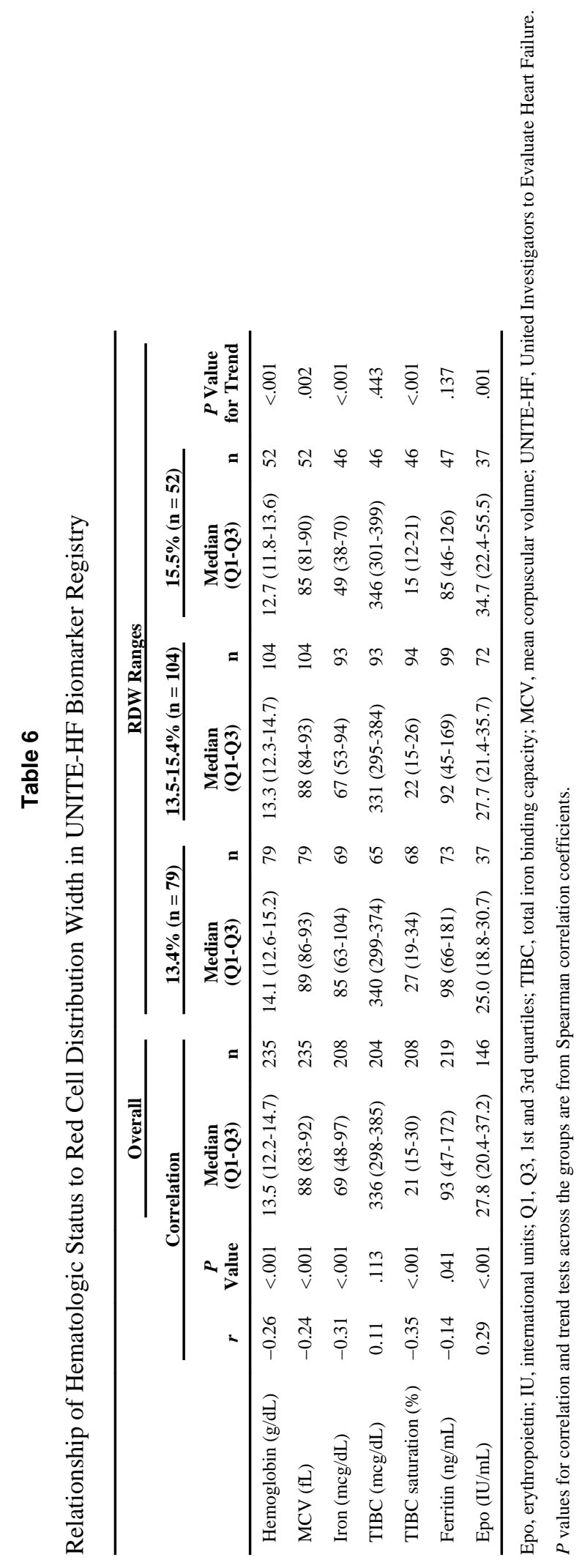


